\title{
BMJ Open Early diagnosis of mortality using admission CT perfusion in severe traumatic brain injury patients (ACT- TBI): protocol for a prospective cohort study
}

\author{
Susan Alcock, ${ }^{1}$ Divjeet Batoo, ${ }^{1}$ Sudharsana Rao Ande, ${ }^{1}$ Rob Grierson, ${ }^{2}$ \\ Marco Essig, ${ }^{1}$ Douglas Martin, ${ }^{2}$ Anurag Trivedi, ${ }^{3}$ Namita Sinha, ${ }^{4}$ Murdoch Leeies, ${ }^{5}$ \\ Frederick A Zeiler (D), 6,7 Jai Jai Shiva Shankar (D) 1,7
}

To cite: Alcock S, Batoo D, Ande $\mathrm{SR}$, et al. Early diagnosis of mortality using admission CT perfusion in severe traumatic brain injury patients (ACT-TBI): protocol for a prospective cohort study. BMJ Open 2021;11:e047305. doi:10.1136/ bmjopen-2020-047305

- Prepublication history and additional online supplemental material for this paper are available online. To view these files, please visit the journal online (http://dx.doi.org/10. 1136/bmjopen-2020-047305)

Received 24 November 2020 Accepted 17 May 2021

Check for updates

(c) Author(s) (or their employer(s)) 2021. Re-use permitted under CC BY-NC. No commercial re-use. See rights and permissions. Published by BMJ.

For numbered affiliations see end of article.

Correspondence to Dr Jai Jai Shiva Shankar; shivajai1@gmail.com

\section{ABSTRACT}

Introduction Severe traumatic brain injury (TBI) is a catastrophic neurological condition with significant economic burden. Early in-hospital mortality ( $<48$ hours) with severe TBI is estimated at $50 \%$. Several clinical examinations exist to determine brain death; however, most are difficult to elicit in the acute setting in patients with severe TBI. Having a definitive assessment tool would help predict early in-hospital mortality in this population. CT perfusion (CTP) has shown promise diagnosing early in-hospital mortality in patients with severe TBI and other populations. The purpose of this study is to validate admission CTP features of brain death relative to the clinical examination outcome for characterizing early inhospital mortality in patients with severe TBI.

Methods and analysis The Early Diagnosis of Mortality using Admission CT Perfusion in Severe Traumatic Brain Injury Patients study, is a prospective cohort study in patients with severe TBI funded by a grant from the Canadian Institute of Health Research. Adults aged 18 or older, with evidence of a severe TBI (Glasgow Coma Scale score $\leq 8$ before initial resuscitation) and, on mechanical ventilation at the time of imaging are eligible. Patients will undergo CTP at the time of first imaging on their hospital admission. Admission CTP compares with the reference standard of an accepted bedside clinical assessment for brainstem function. Deferred consent will be used. The primary outcome is a binary outcome of mortality (dead) or survival (not dead) in the first 48 hours of admission. The planned sample size for achieving a sensitivity of $75 \%$ and a specificity of $95 \%$ with a $\mathrm{Cl}$ of $\pm 5 \%$ is 200 patients.

Ethics and dissemination This study has been approved by the University of Manitoba Health Research Ethics Board. The findings from our study will be disseminated through peer-reviewed journals and presentations at local rounds, national and international conferences. The public will be informed through forums at the end of the study.

Trial registration number NCT04318665

\section{Strengths and limitations of this study}

- This prospective cohort study hopes to improve clinical practice by establishing CT perfusion (CTP) as a diagnostic triage tool which could assist in diagnosing brain death in patients with severe traumatic brain injury (TBI) on admission.

- Our study is the first if its kind where CTP is done at the time of the standard imaging on arrival to hospital.

- This study includes a diversified population of severe TBI patients who are treated at our Provincial Trauma Centre (Health Sciences Centre, Winnipeg).

- Patients receive institutional standard of care after CTP.

- CTP is compared with a reference standard clinical assessment for brainstem function.

\section{INTRODUCTION}

Globally, over 50 million people are estimated to suffer a traumatic brain injury (TBI) annually. ${ }^{1}$ The global economic burden of TBI is estimated at approximately US $\$ 400$ billion per annum. ${ }^{1}$ Primarily affecting healthy individuals with an excellent quality of life, TBI can result in a catastrophic injury, which makes it a huge public health challenge. ${ }^{2}$ In Canada, it is anticipated that TBI will be the most prevalent and costly neurological condition through the year 2031, as it will account for a total indirect cost of over US $\$ 8$ billion. ${ }^{3}$ Furthermore, TBI represents a substantial expenditure for Canadian insurance companies dealing with medical insurance for rehabilitation therapy. ${ }^{3}$

Severity of TBI is classified into mild, moderate, and severe categories using the Glasgow Coma Scale (GCS), with 'severe TBI' defined as a GCS score $\leq 8 .{ }^{3}$ Known as 
the deadly killer or silent epidemic, severe TBI is believed to be the primary cause of postinjury hospitalisation, disability and death throughout the world. ${ }^{34}$ Severe TBI is a life-threatening clinical emergency, during which trauma teams work swiftly in tandem to provide high-level trauma care. Despite very resource-intense care, which may include multiple complicated surgeries, the mortality rate for severe TBI patients within the first 48 hours postadmission remains at $50 \% .{ }^{25}$ Early in-hospital mortality ( $<48$ hours) is likely dependent on the 'preinjury' and more likely on the 'injury' factors. ${ }^{16}$ It is plausible that a significant percentage of these patients have permanent brain damage at the time of hospital admission, including brain death.

\section{Clinical examination for confirmation of brain death}

The clinical examination is the gold standard for diagnosing brain death. ${ }^{7}$ In patients with severe TBI, it can be complicated to elicit because of the nature of the injury itself, and the fact that most patients are on life support and or sedative medications. Therefore, patients often receive elaborate resource-intense treatment despite a lack of clear prognoses. Adding to the quandary of the clinical exam is the fact that there are several outcome scales proposed for the final neurological outcome. ${ }^{1}$ The sheer existence of numerous outcome scales reflects the complexity of long-term outcome assessments in patients with severe TBI. The most used outcome scale is an eightpoint extended Glasgow Outcome Scale (GOSe) ${ }^{8}$

A poor neurological outcome is characterized by a GOSe score of 1 (death), 2 (vegetative state), 3 (severe disability) or 4 (moderate disability). ${ }^{8}$ This outcome assessment is further complicated by preinjury and injury factors as well as patients' response at various stages. The absence of a definitive, reliable, validated, and timely triage tool to predict the higher in-hospital mortality of patients with severe TBI, warrants the urgent need of research in this field. This research may help reduce resource intensive and sometimes futile care in those who are already dead at the time of hospital admission. This may also facilitate the precious gift of organ donation.

\section{Imaging for confirmation of brain death}

Recent systematic reviews and recommendations have found that standard diagnostic imaging techniques (plain CT (CT) and CT angiography (CTA)) currently used to diagnose brain death have inadequate sensitivity. ${ }^{9-11}$ This strengthens the need for advanced techniques to facilitate the early diagnosis of brain death.

Standard of care imaging performed on admission and during the hospital stay of a severe TBI patient is the plain head CT. ${ }^{12}$ Plain CT accurately and promptly diagnoses structural abnormalities but does not provide any functional information including information on brain death. $^{12-17}$

CTA is a readily available, practical, and omnipresent imaging test. However, the CTA protocol for confirmation of brain death varies considerably among different centres, which makes it difficult for it to be accepted as the gold-standard test. ${ }^{18}$ Systematic reviews of the literature have concluded that the sensitivity of CTA is inadequate to confirm brain death and that clinicians should be wary when using it as an ancillary test. ${ }^{9}{ }^{10}$ Moreover, CTA provides anatomic, but not functional, information about the brain. ${ }^{19}$

CT perfusion (CTP) is an advanced CT scan that provides both functional and anatomic information about the brain, and it is mainly used to triage patients with acute stroke. ${ }^{20-25}$ In this technique, the temporal change in contrast density is used to quantify perfusion parameters such as cerebral blood flow (CBF) and cerebral blood volume (CBV), which indicate areas of ischaemic or infarcted brain (areas of 'dead brain'). CTP is very sensitive in detecting $\mathrm{CBF}$ and can detect decreased perfusion as low as $2 \%-3 \%$ in $\mathrm{CBF}$ and $2 \%$ in $\mathrm{CBV}^{26} \mathrm{CBF}$ $<5 \mathrm{~mL} / 100 \mathrm{~g} / \mathrm{min}$ is consistent with clinical diagnosis of brain death. ${ }^{27} 28$ If CTP is unable to detect CBF, or if CTP shows markedly decreased CBF $(<5 \mathrm{~mL} / 100 \mathrm{~g} / \mathrm{min})$, brain death can be confirmed because cellular viability is not possible at such a low CBF. Moreover, CTP with approximately whole-brain coverage can also provide data for CTA.

CTP has rarely been used for patients with severe TBI due to lack of reliable evidence on the efficacy of CTP in this patient population. One of the first uses of CTP in patients with severe TBI on hospital admission, was described by Wintermark $e t a l .^{29} 29$ They found a favourable outcome, which was assessed at 90 days postadmission in patients with normal or high brain perfusion on admission CTP and an unfavourable outcome in patients with low perfusion. Bendinelli $e t a l^{30}$ performed CTP in patients with severe TBI who did not improve neurologically during the first 48 hours after trauma. They found low perfusion in one third of their patients. Additionally, they discovered that CTP altered clinical management in $10 \%$ of their patients who were diagnosed with massive and fatal strokes despite minimal changes on plain CT of head. Of importance, both studies used CTP with limited brain coverage due to technological limitations at the time; this may have missed key findings in other parts of the brain. These two studies addressed the neurological outcome at the end of the hospital stay, but failed to specify any association of CTP results with in-hospital mortality. We were the first to describe the CTP features of brain death as the most sensitive and specific imaging test for confirmation of brain death in intensive care unit (ICU) patients. ${ }^{25}$ Furthermore, we provided the evidence of using CTP to predict in-hospital mortality in comatosed cardiac arrest patients. ${ }^{31}$

A triage tool to facilitate early, if not immediate, decision making is imperative as most deaths occur within 48 hours of hospital admission. ${ }^{1931}$ The first 48 hours of hospitalization involves the most resource-intensive medical and surgical care activities. Some patients with severe TBI may be dead by neurological definition at the time of their hospital admission. Since, an accurate clinical diagnosis 
is obscured by anaesthetic and neuromuscular blockade agents, a validated admission CTP triage tool could assist in diagnosing brain death in these patients. In a small pilot study, our group has shown that CTP could show mortality in $25 \%$ of patients at the time of their hospital admission. ${ }^{32}$ We hypothesize that in severe TBI patients, the CTP scan done at the time of hospital admission, can diagnosis brain death reliably as opposed to the current clinical examination which is routine practice.

The primary objective of the Early Diagnosis of Mortality using Admission CT Perfusion in Severe Traumatic Brain Injury Patients (ACT-TBI) study is to validate admission CTP features of brain death, relative to the clinical outcome of death versus survival, in the first 48 hours of hospital admission, among patients with severe TBI. Secondary objectives include: (1) to establish the safety of admission CTP; (2) to establish the inter-rater reliability of features of brain death on admission CTP.

\section{METHODS AND ANALYSIS}

The ACT-TBI study, is a prospective cohort study in patients with severe TBI, which builds on previous work by our team. ${ }^{32}$ The study design was conceptualized by experts in content (trauma/critical care neurosurgery, neuroradiology, emergency medicine and critical care), epidemiology, and clinical trials to ensure a high-quality study. The ACT-TBI study is under way at the Health Sciences Centre, which is the provincial trauma centre.

\section{Study population}

Our study population of interest are patients with severe TBI who meet the following inclusion criteria: (1) at least 18 years old; (2) severe head injury; (3) GCS score $\leq 8$ after initial resuscitation and (4) on mechanical respiratory ventilation at the time of imaging. Study exclusion criteria include: (1) no known GCS after initial resuscitation; (2) known pregnancy; (3) known contraindication to CT contrast agent, for example, allergy or anaphylactic reaction and (4) known end-stage renal disease.

\section{Patient and public involvement}

Survivors of TBI and their families were involved in the planning, design, recruitment and conduct of the study.

\section{Study Intervention-}

All eligible consecutive patients will be screened and enrolled by the treating emergency department (ED) physician during the initial hospital admission resuscitation, and then undergo a standard imaging protocol, (whole body CT scan, including the plain head CT) as well as the study CT-perfusion protocol of the whole head (figure 1). Images will be acquired following our previously published protocol. ${ }^{32}$ In brief, a total of $40 \mathrm{~mL}$ of CT contrast media will be injected at a rate of $5 \mathrm{~mL} / \mathrm{s}$. A set of axial images with a slice thickness of $5 \mathrm{~mm}$ for the perfusion analysis will be reconstructed. All patients will receive standard of care post imaging and the results of the CTP

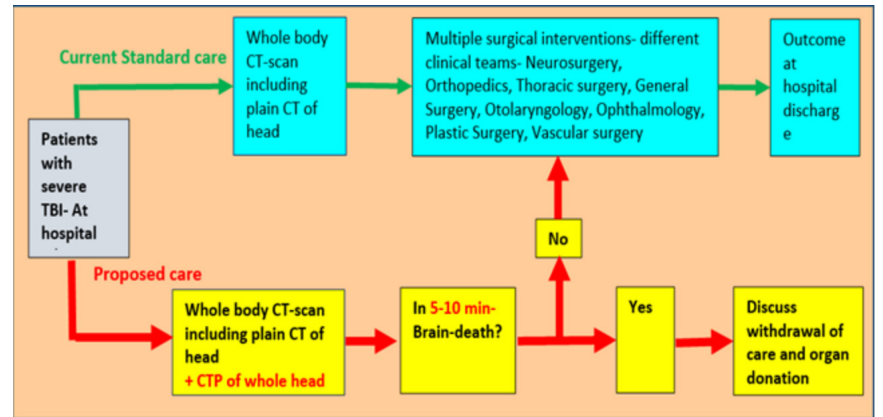

Figure 1 Illustration of the current hospital standard of care workflow (in blue) for severe TBI patients at admission and the intervention from our research protocol (in yellow). CTP, CT perfusion; TBI, traumatic brain injury.

will not be made available to the clinical team involved in patient care, since the prognostic value of CTP has not been established in this patient population.

\section{Retention strategies}

At our centre, one universal requisition is used for all diagnostic imaging tests. To improve study adherence and to remind the ED team of the study, a red stamp was placed on each requisition in the department (see figure 2). Furthermore, at the end of each month, the ED team and the Diagnostic Imaging team receive an email indicating the study's enrolment status, and a brief reminder of the study and its objectives. To facilitate the study's recruitment goal, the study team monitors the provincial diagnostic database system daily for CTP scans. A log of the screened patients is then kept by the study coordinator. The study design and intervention do not require significant changes to current standard clinical practice, as the addition of CTP images is the only change in practice. No issues are foreseen in data collection.

\section{Timeline}

We plan to complete the ACT-TBI study within 2 years. Study preparation including clinical research documentation, institutional ethics committee approvals, training of research staff involved in the study, and pilot validation took approximately 6 months. Ethics approval was obtained on 24 February, 2020. Actual patient enrolment began on 23 July, 2020. Most trauma centres receive a minimum of five patients with severe TBI per week. With a conservative enrolment rate of approximately 15-17 patients per month, the enrolment should be accomplished within 1year. Data preparation including the CTP image analysis will commence in the final year of the study. A description of the study timeline can be found in table 1 .

\section{Data Collection and Management \\ Clinical data}

Clinical data will be collected by a qualified data abstractor with knowledge of the study. A qualified data abstractor will collect data from the patient's chart using a chart abstraction tool. The case report form for our study can 
Traumatic Brain Injury:

$\begin{array}{lll}\text { Head Injury } & \text { Yes } & \text { No } \\ \text { GCS } \leq 8 & \text { Yes } & \text { No }\end{array}$

Figure 2 Study stamp for the diagnostic imaging requisition.

be found in online supplemental appendix A. Existing injuries will be documented using the new Injury Severity Scale. ${ }^{33}$ Process metrics such as, the number of surgical interventions on each patient, the length of stay in ICU, and the hospital length of stay will be derived from the data collected. All clinical data will be entered into an Excel database. Clinical Data cleaning and validation will be completed by a master's student. The principal investigator will ensure quality assurance policies are being implemented to confirm study data are reported in compliance with the protocol, which include: reviewing the screening logs; monitoring of recruitment trends; reviewing the data collected on the case report forms; generating queries regarding outliers or missing data and implementing data tracking procedures.

\section{Imaging data}

CTP images will be acquired at the time of admission. An overview of the imaging data collected for our study can be found in online supplemental appendix A. The anonymized images will be transferred and stored in the secured imaging core lab in the department of Radiology at the University of Manitoba. The imaging centre (Department of Radiology, University of Manitoba, Winnipeg) is responsible for the postprocessing of the CTP raw data, image interpretation and image analysis. CTP will be processed using a semiautomatic deconvolution algorithm on a vendor neutral software package (Oleasphere). CTP will be assessed quantitatively such that brain death will be defined as $\mathrm{CBF}<5 \mathrm{~mL} / 100 \mathrm{~g} / \mathrm{min}$ and $\mathrm{CBV}<2 \mathrm{~mL} / 100 \mathrm{~g}$ in the brainstem. CTP will also be assessed qualitatively with brain death being defined as

\begin{tabular}{l} 
Table 1 Description of Timeline for the ACT-TBI study \\
\hline \multicolumn{1}{c}{ Year 1} \\
\hline Protocol and CRF finalisation \\
REB submissions and approvals \\
Start-up meeting and training \\
Site activation \\
Screening and enrolment \\
Data collection \\
Steering committee meetings \\
Adjudication \\
Data preparation and analyses \\
Dissemination \\
\hline
\end{tabular}

ACT-TBI, Early Diagnosis of Mortality using Admission CT Perfusion in Severe Traumatic Brain Injury Patients; CRF, clinical record form; REB, research ethics board. a matched decrease of $\mathrm{CBF}$ and $\mathrm{CBV}$ in the brainstem. During the first 48 hours of hospital admission and at the end of the patients hospital stay, the perfusion maps for $\mathrm{CBF}$ and $\mathrm{CBV}$ will be assessed for the binary outcome of 'dead' or 'not-dead', according to our previously published methods. ${ }^{41-34}$ The perfusion images will be reviewed by two-independent neuroradiologists, who will be blinded to the clinical status of the patient and also to each other's assessment. In case of disagreement, a consensus agreement will be employed for the final analysis.

\section{Data analysis and power considerations}

The gold standard for diagnosis of brain death is confirmation by clinical examination. Sensitivity is defined as the ability of CTP to correctly classify an individual as 'dead', while ability of CTP to correctly classify an individual as 'not-dead' is a specificity. Positive predictive value (PPV) is the percentage of patients showing features of brain death on CTP, who are clinically 'dead'. Negative predictive value (NPV) is the percentage of patients with no features of brain death on CTP, who were clinically 'not-dead'. An ideal triage test for confirmation of brain death should have no false positives, that is, calling someone 'dead' when they are not. Thus, an ideal triage test should have $100 \%$ specificity and PPV. In our pilot study, CTP showed $100 \%$ specificity and PPV as well as $75 \%$ sensitivity and $94 \%$ NPV for correctly classifying in-hospital mortality. Based on these preliminary results by employing the Buderer's formula, ${ }^{35}$ we calculated a sample size for the ACT-TBI study. A total of 180 patients with severe TBI (with a conservative prevalence of in-hospital mortality of at least $40 \%$ ) will be required to validate the features of brain death on admission CTP against in-hospital mortality. This will allow us to achieve a sensitivity of at least $75 \%$ and specificity of $95 \%$ with a CI $\pm 5 \%$ around the point estimate. Since the study endpoint is at hospital discharge, we do not expect any loss to follow-up. With a plausible drop-out of $10 \%$ (including technical problems with CTP acquisition, protocol violation, consent withdrawal or possible contraindication for CTP), the sample size was increased by an absolute number of 20. Thus, a total of 200 patients with severe TBI will be targeted to enrol in the ACT-TBI study.

Baseline characteristics of patients will be described using frequency distribution statistics and measure of dispersion. The primary validity analysis will be performed using sensitivity, specificity, PPV and NPV for features of brain death on admission CTP compared with the in-hospital mortality along with $95 \%$ CI. Area under the receiver operating characteristics curve will be calculated to characterize the diagnostic ability of brain death features on CTP for in-hospital mortality. Interobserver agreement between two neuroradiologists will be assessed using the kappa statistic to assess the reliability of CTP. Logistic regression will be used to build predictive models for clinical outcome at discharge for admission CTP, IMPACT variables, ${ }^{36}$ and other standard baseline variables such 
as age, sex, baseline GCS score, nature of the baseline head injury. The predictive models will be used to calculate the area under the receiver operating characteristics curve to enable comparison of the models. Complications including renal failure or allergic reactions associated with CTP will be reported in percentage.

\section{DISCUSSION}

Outcomes of the ACT-TBI study will validate the admission CTP as a pioneer triage tool, thereby offering improved diagnosis and prognosis for brain death in patients with severe TBI, which can be further confirmed by the clinical examination gold standard. Adopting this triage tool in the routine practice to evaluate patients with severe TBI will help reduce the use of resource intensive and sometimes futile treatment to potentially dead patients in the emergency room. Furthermore, using CTP in this setting will improve trust and investment in organ transplantation in Canada and worldwide.

One study limitation is that our prospective study is a single centre study; this may affect its generalisability. Our prospective study, however, has rigour in that the study intervention is controlled and the outcome measures are preselected. ACT-TBI study is the first of its kind where admission CTP will be used to assess mortality in patients with severe TBI at the time of hospital admission. Besides this vital objective, we also planned two nested studies: (1) to examine the imaging biomarkers as predictors of functional outcome in patients with severe TBI and pathological correlation of the imaging biomarkers on autopsy, when possible and on receiving the separate consent and (2) health economic benefits of employing admission CTP.

\section{ETHICS AND DISSEMINATION}

We have received ethics approval from the University of Manitoba Health Research Ethics Board number-HS23683- (B2020:018) and the study is being conducted in compliance with Good Clinical Practices on Ethical Conduct for Research Involving Humans.

Deferral of consent has been approved by our institutional ethics board. Considering the altered level of the participant's consciousness, informed consent will be obtained from a substitute legal decision-maker by deferred consent within 1 week of enrolment. Informed consent will be obtained by the site research coordinator/ research nurse.

All data collected for the study will remain strictly confidential. Anonymity of the study participants is addressed by using case numbers. A record of the patient's ID and case number is being kept in a separate secure passwordprotected file accessible only by the study coprincipal investigators and study coordinator. Data will be presented in an aggregate form only. Data collection forms will be stored for at least 10 years.
The dissemination plan includes the traditional dissemination vehicles (eg, presentations at local grand rounds, national and international conferences, publications of studies in open-access peer-reviewed journals). Once proven useful, admission CTP will be incorporated in the clinical guidelines for imaging in patients with severe TBI. This will bring the evidence-based clinical practice change at the national and international level. The knowledge translation plan will also employ other approaches that will aim to incorporate the findings into the design of future studies. A Café Scientifique session at the end of the study will be organized to inform the general public about the results of our study and how it will influence future clinical practice. In completing the study monitoring activities, we will be conducting a type of ongoing general quality improvement in the care of patients with severe TBI. This will function as a mechanism of integrative knowledge translation that will ultimately be transferable to implementation of this treatment in routine clinical practice.

\section{Author affiliations}

${ }^{1}$ Department of Radiology, University of Manitoba Faculty of Health Sciences, Winnipeg, Manitoba, Canada

${ }^{2}$ Department of Emergency Medicine, University of Manitoba Faculty of Health Sciences, Winnipeg, Manitoba, Canada

${ }^{3}$ Section of Neurology, Department of Internal Medicine, University of Manitoba Faculty of Health Sciences, Winnipeg, Manitoba, Canada

${ }^{4}$ Section of Neuropathology, Department of Pathology, University of Manitoba Faculty of Health Sciences, Winnipeg, Manitoba, Canada

${ }^{5}$ Department of Emergency Medicine \& Section of Critical Care Medicine, University of Manitoba Faculty of Health Sciences, Winnipeg, Manitoba, Canada

${ }^{6}$ Section of Neurosurgery, Department of Surgery, University of Manitoba Faculty of Health Sciences, Winnipeg, Manitoba, Canada

${ }^{7}$ Department of Human Anatomy and Cell Science, University of Manitoba Faculty of Health Sciences, Winnipeg, Manitoba, Canada

Acknowledgements We are grateful to all the patients that participated in the study and would like to thank the Health Sciences Centre Adult Emergency for their involvement in subject recruitment and the CT technologists for performing the CT perfusion scans. We would also like to thank Cecelia and Steven Lipischak for their letter of support for our grant application, which was through the lens of a family member and patient.

Contributors JJSS, FAZ, RG, ME, DM, AT, NS and SRA contributed to the conception and design of the study, provided input into the protocol, and gave critical feedback on the manuscript. JJSS, SA and DB obtain consents. SA and DB perform study coordinator duties and collect clinical data. DB collects the imaging data. ML provides ongoing study support within the ED. JJSS, FAZ, RG, ML, SA and $\mathrm{DB}$ attend quarterly ACT-TBI steering committee meetings. SA wrote the initial draft of the manuscript. All authors approved the final version of the manuscript.

Funding This work is supported by the Canadian Institute of Health Research, grant number 201909PJM-432061-BSB-CDAA-283730. JJSS receives salary research support award from Radiology consultants of Winnipeg. FAZ receives research support from the Manitoba Public Insurance (MPI) Neuroscience/TBI Research Endowment, the Health Sciences Centre Foundation Winnipeg, the United States National Institutes of Health (NIH) through the National Institute of Neurological Disorders and Stroke (NINDS)(Grant \#: R03NS114335-01), the Canadian Institutes of Health Research (CIHR)(Grant \#: 432061), the Canada Foundation for Innovation (CFI)(Project \#: 38583), Research Manitoba (Grant \#: 3906), the University of Manitoba VPRI Research Investment Fund (RIF), the University of Manitoba Centre on Aging and the University of Manitoba Rudy Falk Clinician-Scientist Professorship.

Competing interests None declared.

Patient consent for publication Not required. 
Provenance and peer review Not commissioned; externally peer reviewed.

Supplemental material This content has been supplied by the author(s). It has not been vetted by BMJ Publishing Group Limited (BMJ) and may not have been peer-reviewed. Any opinions or recommendations discussed are solely those of the author(s) and are not endorsed by BMJ. BMJ disclaims all liability and responsibility arising from any reliance placed on the content. Where the content includes any translated material, BMJ does not warrant the accuracy and reliability of the translations (including but not limited to local regulations, clinical guidelines, terminology, drug names and drug dosages), and is not responsible for any error and/or omissions arising from translation and adaptation or otherwise.

Open access This is an open access article distributed in accordance with the Creative Commons Attribution Non Commercial (CC BY-NC 4.0) license, which permits others to distribute, remix, adapt, build upon this work non-commercially, and license their derivative works on different terms, provided the original work is properly cited, appropriate credit is given, any changes made indicated, and the use is non-commercial. See: http://creativecommons.org/licenses/by-nc/4.0/.

\section{ORCID iDs}

Frederick A Zeiler http://orcid.org/0000-0003-1737-0510

Jai Jai Shiva Shankar http://orcid.org/0000-0002-3707-9723

\section{REFERENCES}

1 National Collaborating Centre for Acute Care (UK). Head injury: triage, assessment, investigation and early management of head injury in infants, children and adults. London National Collaborating Centre for Acute Care (UK); 2007.

2 Shankar JJS, Vandorpe R. CT perfusion for confirmation of brain death. AJNR Am J Neuroradiol 2013;34:1175-9.

3 Narayan RK, Michel ME, Ansell B, et al. Clinical trials in head injury. $J$ Neurotrauma 2002;19:503-57.

4 Evans JA, van Wessem KJP, McDougall D, et al. Epidemiology of traumatic deaths: comprehensive population-based assessment. World J Surg 2010;34:158-63.

5 MacDonald D, Stewart-Perrin B, Shankar JJS. The role of neuroimaging in the determination of brain death. $J$ Neuroimaging 2018;28:374-9.

6 McMillan T, Bigler ED, Teasdale G, et al. Outcome assessment after traumatic brain injury. Lancet Neurol 2018;17:299.

7 Shemie SD, Hornby L, Baker A, et al. International Guideline development for the determination of death. Intensive Care Med 2014;40:788-97.

8 Wilson JT, Pettigrew LE, Teasdale GM. Structured interviews for the Glasgow outcome scale and the extended Glasgow outcome scale: guidelines for their use. J Neurotrauma 1998;15:573-85.

9 Kramer $\mathrm{AH}$, Roberts DJ. Computed tomography angiography in the diagnosis of brain death: a systematic review and meta-analysis. Neurocrit Care 2014;21:539-50.

10 Taylor T, Dineen RA, Gardiner DC, et al. Computed tomography (CT) angiography for confirmation of the clinical diagnosis of brain death. Cochrane Database Syst Rev 2014:CD009694.

11 Wintermark M, Sanelli PC, Anzai Y, Tsiouris A, et al. Imaging evidence and recommendations for traumatic brain injury: advanced neuroand neurovascular imaging techniques. AJNR Am J Neuroradiol 2015;36:E1-11.

12 Mayer SA, Kossoff SB. Withdrawal of life support in the neurological intensive care unit. Neurology 1999;52:1602.

13 Corral L, Herrero JI, Monfort JL, et al. First CT findings and improvement in GOS and GOSE scores 6 and 12 months after severe traumatic brain injury. Brain Inj 2009;23:403-10.

14 Huang AP-H, Lee C-W, Hsieh H-J, et al. Early parenchymal contrast extravasation predicts subsequent hemorrhage progression, clinical deterioration, and need for surgery in patients with traumatic cerebral contusion. J Trauma 2011;71:1593-9.
15 Mushkudiani NA, Hukkelhoven CWPM, Hernández AV, et al. A systematic review finds methodological improvements necessary for prognostic models in determining traumatic brain injury outcomes. $J$ Clin Epidemiol 2008;61:331-43.

16 Soustiel JF, Mahamid E, Goldsher D, et al. Perfusion-CT for early assessment of traumatic cerebral contusions. Neuroradiology 2008;50:189-96.

17 Astrup J. Energy-Requiring cell functions in the ischemic brain. their critical supply and possible inhibition in protective therapy. $J$ Neurosurg 1982;56:482-97.

18 Greer DM, Varelas PN, Haque S, et al. Variability of brain death determination guidelines in leading US neurologic institutions. Neurology 2008;70:284-9.

19 Shankar JJS, Stewart-Perrin B, Quraishi A-U-R, et al. Computed tomography perfusion AIDS in the prognostication of comatose postcardiac arrest patients. Am J Cardiol 2018;121:874-8.

20 Shankar JJS, Langlands G, Doucette S, et al. Ct perfusion in acute stroke predicts final infarct volume- inter-observer study. Can $J$ Neurol Sci 2016;43:93-7.

21 Wilson CD, Shankar JJS. Diagnosing vasospasm after subarachnoid hemorrhage: cta and CTP. Can J Neurol Sci 2014;41:314-9.

22 Shankar JJS, Lum C. Whole brain CT perfusion on a 320-slice CT scanner. Indian J Radiol Imaging 2011;21:209-14.

23 Nogueira RG, Jadhav AP, Haussen DC, et al. Thrombectomy 6 to 24 hours after stroke with a mismatch between deficit and infarct. $N$ Engl J Med 2018;378:11-21.

24 Albers GW, Lansberg MG, Kemp S, et al. A multicenter randomized controlled trial of endovascular therapy following imaging evaluation for ischemic stroke (DEFUSE 3). Int J Stroke 2017;12:896-905.

25 Du EHY, Shankar JJS. Rapid endovascular treatment of acute ischemic stroke: what a general radiologist should know. Can Assoc Radiol J 2017;68:154-60.

26 Uwano I, Kudo K, Sasaki M, et al. CT and MR perfusion can discriminate severe cerebral hypoperfusion from perfusion absence: evaluation of different commercial software packages by using digita phantoms. Neuroradiology 2012;54:467-74.

27 Pistoia F, Johnson DW, Darby JM, et al. The role of xenon CT measurements of cerebral blood flow in the clinical determination of brain death. AJNR Am J Neuroradiol 1991;12:97-103.

28 Thompson JR, Ashwal S, Schneider S, et al. Comparison of cerebral blood flow measurements by xenon computed tomography and dynamic brain scintigraphy in clinically brain dead children. Acta Radiol Suppl 1986;369:675-9.

29 Wintermark M, van Melle G, Schnyder P, et al. Admission perfusion $\mathrm{CT}$ : prognostic value in patients with severe head trauma. Radiology 2004;232:211-20.

30 Bendinelli C, Bivard A, Nebauer S, et al. Brain CT perfusion provides additional useful information in severe traumatic brain injury. Injury 2013;44:1208-12.

31 Trauma registry report on injury in nova Scotia: provincial report 2012-2013 data year. 2014.

32 Shankar JJS, Green R, Virani K, et al. Admission perfusion CT for classifying early in-hospital mortality of patients with severe traumatic brain injury: a pilot study. AJR Am J Roentgenol 2020;214:872-6.

33 Stevenson M, Segui-Gomez M, Lescohier I, et al. An overview of the injury severity score and the new injury severity score. Inj Prev 2001;7:10-13.

34 National Scientific Advisory Committee, Canadian Institute of Health Research. Identifying priorities for research and capacity development in injury as a Multi-Institute strategic initiative; 2004.

35 Buderer NM. Statistical methodology: I. incorporating the prevalence of disease into the sample size calculation for sensitivity and specificity. Acad Emerg Med 1996;3:895-900.

36 Steyerberg EW, Mushkudiani N, Perel P, et al. Predicting outcome after traumatic brain injury: development and international validation of prognostic scores based on admission characteristics. PLoS Med 2008;5:e165. 